\title{
On the Chebyshev property of certain Abelian integrals near a polycycle
}

\author{
D. Marín and J. Villadelprat \\ Departament de Matemàtiques, Facultat de Ciències, \\ Universitat Autònoma de Barcelona, 08193 Bellaterra, Barcelona, Spain \\ Departament d'Enginyeria Informàtica i Matemàtiques, ETSE, \\ Universitat Rovira i Virgili, 43007 Tarragona, Spain
}

\begin{abstract}
F. Dumortier and R. Roussarie formulated in [Birth of canard cycles, Discrete Contin. Dyn. Syst. 2 (2009) 723-781] a conjecture concerning the Chebyshev property of a collection $I_{0}, I_{1}, \ldots, I_{n}$ of Abelian integrals arising from singular perturbation problems occurring in planar slow-fast systems. The aim of this note is to show the validity of this conjecture near the polycycle at the boundary of the family of ovals defining the Abelian integrals. As a corollary of this local result we get that the linear span $\left\langle I_{0}, I_{1}, \ldots, I_{n}\right\rangle$ is Chebyshev with accuracy $k=k(n)$.
\end{abstract}

\section{Introduction and statement of the main result}

This paper is concerned with the problem of studying when a collection of Abelian integrals form an extended complete Chebyshev system (see Definition 2.1). This type of problem arises in the context of the so-called infinitesimal Hilbert's 16th problem proposed by Arnold [1]. In the present paper we are interested in a conjecture formulated by Dumortier and Roussarie in [4], where the authors consider singular perturbation problems occurring in planar slow-fast systems depending on parameters. They investigate the number of limit cycles that appear near a slow-fast Hopf point, i.e., its cyclicity. Their main results show that under very general conditions this cyclicity is finite and, modulo the aforementioned conjecture, provide its sharp upper bound. In order to give a precise statement of their conjecture let us consider the function $H: \mathbb{R}^{2} \longrightarrow \mathbb{R}$ given by $H(x, y)=e^{-x}\left(1+x-\frac{1}{2} y^{2}\right)$. It can be checked that the level sets $\{H(x, y)=h\}$ for $h \in(0,1)$ are ovals $\gamma_{h}$ surrounding the origin. The family $\left\{\gamma_{h}\right\}_{h \in(0,1)}$ form a period annulus and its boundary has two connected components, the parabola $y^{2}=2(x+1)$ and the origin $(0,0)$, which are the level sets $h=0$ and $h=1$, respectively (see Figure 1). Let us define the family of Abelian integrals

$$
I_{k}(h):=\int_{\gamma_{h}} y^{2 k-1} d x, \quad k \in \mathbb{Z}^{+} .
$$

With this notation, the conjecture posed by Dumortier and Roussarie in [4] is the following:

Conjecture. For each $n \geqslant 0,\left(I_{0}, I_{1}, \ldots, I_{n}\right)$ is an ECT-system on $\left[h_{0}, 1\right]$ for any $h_{0} \in(0,1)$.

The validity of this conjecture for $n \leqslant 2$ is proved in [5]. Moreover, taking advantage of the analyticity of $I_{k}$ at $h=1$, in that paper is also proved that for each $n \geqslant 0$ there exists $\varepsilon>0$ such that $\left(I_{0}, I_{1}, \ldots, I_{n}\right)$ is an ECT-system on $(1-\varepsilon, 1]$, see [5, Corollary 3.5]. In other words, that the conjecture is true near the

Keywords: Chebyshev systems, Abelian integrals, Wronskian.

2010 MSC: $34 \mathrm{C}, 41 \mathrm{~A}$.

Marín is partially supported by MTM2015-66165-P and Villadelprat is partially supported by MTM2014-52209-C2-1-P. 
center. The aim of the present paper is to show the same property near the polycycle. More concretely our main result is the following:

Theorem A. For each $n \geqslant 0$ there exists $\varepsilon>0$ such that $\left(I_{0}, I_{1}, \ldots, I_{n}\right)$ is an ECT-system on $(0, \varepsilon)$.

Besides giving another evidence in support of the validity of the conjecture of Roussarie and Dumortier, Theorem A also implies that for each $n \geqslant 0$ there exists a non-negative integer $k=k(n)$ such that the linear span $\left\langle I_{0}, I_{1}, \ldots, I_{n}\right\rangle$ is Chebyshev with accuracy $k$ in $(0,1]$, see Corollary 2.7. In this regard note that the validity of the conjecture is equivalent to $k(n)=0$ for all $n \geqslant 0$. For other results about the analytic properties of the Abelian integrals $I_{k}(h)$ the reader is referred to the paper of Françoise and Xiao [6].

The proof of Theorem A is based on an accurate study of the asymptotic properties of the Abelian integrals $I_{k}(h)$ at $h=0$, which in the Poincaré disc is a polycycle. In the literature (see [8] and references there in) there are results that provide the expression of the coefficients in the asymptotic development of Abelian integrals near certain polycycles. However we cannot apply them because in our case the singular point at the polycycle is very degenerated. We bypass this problem by composing the Abelian integral with an infinitely flat function. Although this is certainly an ad hoc solution we hope that it can be adapted to tackle similar problems. That being said, let us stress that by no means the cyclicity of the polycycle can be deduced from Theorem A. An upper bound for the cyclicity is proved in [4], see Theorem 5.11, and the authors mention that it is only one unit higher than the sharp upper bound that they expect to hold.

\section{Proof of the main result}

Definition 2.1. Let $f_{0}, f_{1}, \ldots f_{n-1}$ be analytic functions on an interval $I$. The ordered set $\left(f_{0}, f_{1}, \ldots f_{n-1}\right)$ is an extended complete Chebyshev system (for short, an ECT-system) on $I$ if, for all $k=1,2, \ldots n$, any nontrivial linear combination

$$
\alpha_{0} f_{0}(x)+\alpha_{1} f_{1}(x)+\cdots+\alpha_{k-1} f_{k-1}(x)
$$

has at most $k-1$ isolated zeros on $I$ counted with multiplicities.

To prove Theorem A we first need to show several technical lemmas dealing with some particular integrals. For reader's convenience we advance the idea of the proof in order to understand the role of these integrals. To this end note that, for each $h \in(0,1)$, the oval $\gamma_{h}$ intersects the $x$-axis at two points $\left(x_{ \pm}(h), 0\right)$, where $x_{-}(h)<0<x_{+}(h)$ are the roots of $f(x):=e^{-x}(1+x)=h$. Thus, since $y^{2}=2\left(1+x-h e^{x}\right)$ for all $(x, y) \in \gamma_{h}$, we can split each Abelian integral as

$$
I_{k}(h)=2^{k+\frac{1}{2}} \underbrace{\int_{x_{-}(h)}^{0}\left(1+x-h e^{x}\right)^{k-\frac{1}{2}} d x}_{I_{k}^{-}(h)}+2^{k+\frac{1}{2}} \underbrace{\int_{0}^{x_{+}(h)}\left(1+x-h e^{x}\right)^{k-\frac{1}{2}} d x}_{I_{k}^{+}(h)} .
$$

Note that in principle $x_{ \pm}(h)$ are well-defined analytic functions for $h \in(0,1)$. However, due to $f^{\prime}(-1) \neq 0$, it follows that $x_{-}(h)$ can be extended analytically to $h=0$ by setting $x_{-}(0)=-1$. This will be a key point in our proof because it shows that, contrary to $I_{k}^{+}$, the function $I_{k}^{-}$is analytic at $h=0$. To take advantage of this we will compose $I_{k}$ with a diffeomorphism which is infinitely flat at $h=0$. More concretely, we perform the change of variable given by $h=f(1 / s)=e^{-1 / s}(1+1 / s)$ and define

$$
\hat{I}_{k}(s):=I_{k}(f(1 / s)) \text { for any } s>0 .
$$

Since $s \longmapsto f(1 / s)$ is an analytic diffeomorphism from $(0,+\infty)$ to $(0,1)$ with $\lim _{s \rightarrow 0^{+}} f(1 / s)=0$, it is clear that proving Theorem $\mathrm{A}$ is equivalent to show that for each $n \geqslant 0$ there exists $\varepsilon>0$ such that $\left(\hat{I}_{0}, \hat{I}_{1}, \ldots, \hat{I}_{n}\right)$ is an ECT-system on $(0, \varepsilon)$. With this aim in view we also define

$$
\hat{I}_{k}^{-}(s):=I_{k}^{-}(f(1 / s)) \text { and } \hat{I}_{k}^{+}(s):=I_{k}^{+}(f(1 / s)) \text {. }
$$




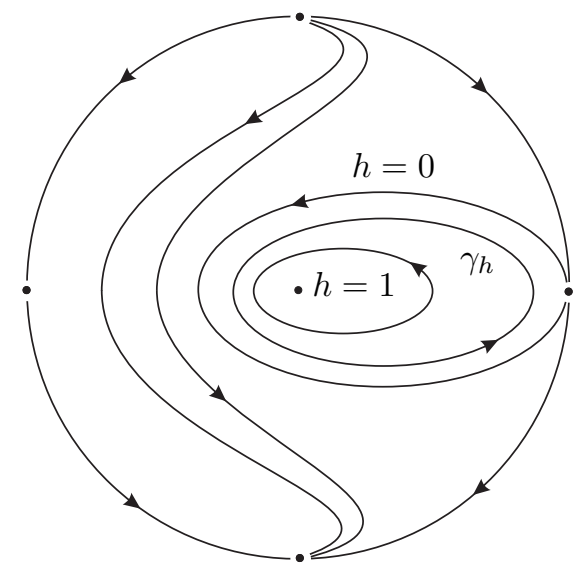

Figure 1: Phase portrait in the Poincaré disc of the differential system $-y \partial_{x}+\left(x-\frac{1}{2} y^{2}\right) \partial_{y}$, which has $H(x, y)=e^{-x}\left(1+x-\frac{1}{2} y^{2}\right)$ as a first integral.

The technical lemmas that we mentioned before are concerned with the behaviour of $\hat{I}_{k}^{+}(s)$ as $s \longrightarrow 0^{+}$. In the statement of the first one we use the notation

$$
\psi_{\alpha}(s):=\int_{0}^{1}\left((1+s)\left(1-e^{-\frac{t}{s}}\right)-t\right)^{\alpha} d t, \text { for } \alpha>-1 .
$$

Lemma 2.2. $\hat{I}_{k}^{+}(s)=s^{-\left(k+\frac{1}{2}\right)} \psi_{k-\frac{1}{2}}(s)$ for all $s>0$.

Proof. By definition $x_{+}(h)$ is the positive solution of $f(x)=h$. Accordingly $x_{+}(f(s))=s$ for all $s>0$. Taking this into account and performing the change of variable $x=(1-t) / s$ we get

$$
\hat{I}_{k}^{+}(s)=I_{k}^{+}(f(1 / s))=\int_{0}^{\frac{1}{s}}\left(1+x-(1+1 / s) e^{x-\frac{1}{s}}\right)^{k-\frac{1}{2}} d x=s^{-\left(k+\frac{1}{2}\right)} \int_{0}^{1}\left((1+s)\left(1-e^{-\frac{t}{s}}\right)-t\right)^{k-\frac{1}{2}} d t,
$$

and this proves the result.

Next, setting

$$
J_{\alpha}(s):=\int_{0}^{1-e^{1-\frac{1}{s}}}(x+s \log (1-x))^{\alpha} d x, \text { for } s>0,
$$

we get the following identity.

Lemma 2.3. $\psi_{\alpha}(s)=\frac{-1}{\alpha+1}\left((1+s)\left(1-e^{-\frac{1}{s}}\right)-1\right)^{\alpha+1}+(1+s)^{\alpha+1} J_{\alpha}\left(\frac{s}{1+s}\right)$.

Proof. Performing the change of variable $x=1-e^{-\frac{t}{s}}$ in (2) we get

$$
\psi_{\alpha}(s)=\int_{0}^{1-e^{-1 / s}}((1+s) x+s \log (1-x))^{\alpha} \frac{s d x}{1-x} .
$$

Next we make the change $u=(1+s) x+s \log (1-x)$, which on account of $\frac{s}{1-x} d x=(1+s) d x-d u$ yields

$$
\begin{aligned}
\psi_{\alpha}(s) & =-\int_{0}^{(1+s)\left(1-e^{-1 / s}\right)-1} u^{\alpha} d u+(1+s) \int_{0}^{1-e^{-1 / s}}((1+s) x+s \log (1-x))^{\alpha} d x \\
& =\frac{-1}{\alpha+1}\left((1+s)\left(1-e^{-\frac{1}{s}}\right)-1\right)^{\alpha+1}+(1+s)^{\alpha+1} \int_{0}^{1-e^{-1 / s}}\left(x+\frac{s}{1+s} \log (1-x)\right)^{\alpha} d x .
\end{aligned}
$$




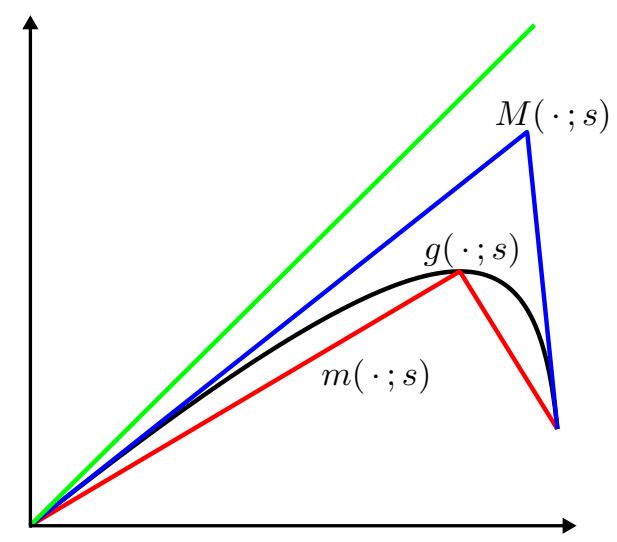

Figure 2: The graphs $g(\cdot ; s), m(\cdot ; s)$ and $M(\cdot ; s)$ for $s=0.2$. The three functions tend to the identity (in green) as $s \longrightarrow 0^{+}$.

From this equality, an easy computation shows the validity of the result.

Lemma 2.4. For every $\alpha>-1, \lim _{s \rightarrow 0^{+}} J_{\alpha}(s)=\frac{1}{1+\alpha}$. Moreover $\lim _{s \rightarrow 0^{+}} s^{\ell} \partial_{s}^{\ell} J_{\alpha}(s)=0$ for all $\ell \in \mathbb{N}$.

Proof. Let us set $\phi(s):=e^{1-1 / s}$, which is a flat function at $s=0$, and $g(x ; s):=x+s \log (1-x)$. Then we can write $J_{\alpha}(s)=\int_{0}^{1-\phi(s)} g^{\alpha}(x ; s) d x$. One can easily verify that, for each $s>0$, the function $x \longmapsto g(x ; s)$ is convex and has a maximum at $x_{s}:=1-s$ with $g\left(x_{s} ; s\right)=1+s(\log s-1)$. We take advantage of this in order to bound $g(\cdot ; s)$ on $[0,1-\phi(s)]$ between two piecewise linear functions. To this end let us define

$$
c_{-}(s):=\frac{1-s+s \log s}{1-s}, \quad c_{+}(s):=1-s, \omega(s):=\frac{1+s(\log s-2)+\phi(s)}{1-\phi(s) / s} \text { and } \varphi(s):=\frac{1-s}{1-\phi(s)} \frac{\phi(s)}{s} .
$$

Observe that $c_{ \pm}(s)$ and $\omega(s)$ tend to 1 as $s \longrightarrow 0^{+}$and that $\varphi(s)$ is flat at $s=0$. Then, for each fixed $s>0$, $m(x ; s) \leqslant g(x ; s) \leqslant M(x ; s)$ for all $x \in[0,1-\phi(s)]$, where

$$
m(x ; s):= \begin{cases}c_{-}(s) x & \text { if } x \in[0,1-s], \\ \frac{\omega(s)}{s}(1-\phi(s)-x)+s-\phi(s) & \text { if } x \in[1-s, 1-\phi(s)],\end{cases}
$$

and

$$
M(x ; s):= \begin{cases}c_{+}(s) x & \text { if } x \in[0,1-\varphi(s)] \\ (1-s / \phi(s))(x-1) & \text { if } x \in[1-\varphi(s), 1-\phi(s)] .\end{cases}
$$

Indeed, the fact that $M(\cdot ; s)$ is an upper bound for $g(\cdot ; s)$ follows from the convexity of the latter and that, by construction, $\partial_{x} m(0 ; s)=\partial_{x} g(0 ; s)$ and $\partial_{x} m(1-\phi(s) ; s)=\partial_{x} g(1-\phi(s) ; s)$, see Figure 2. Similarly, since $g(\cdot ; s)$ is a convex function with a maximum at $x_{s}$ and, by construction, $m\left(x_{s} ; s\right)=g\left(x_{s} ; s\right)$, one can readily see that $m(\cdot ; s)$ is a lower bound.

On the other hand one can easily show by induction on $\ell \geqslant 0$ that, on account of $\phi^{\prime}(s)=\phi(s) / s^{2}$,

$$
\partial_{s}^{\ell} J_{\alpha}(s)=P_{\ell}(s, \phi(s))\left(1-\frac{\phi(s)}{s}\right)^{\alpha-\ell+1} \frac{\phi(s)}{s^{3 \ell-\alpha-1}}+\kappa_{\alpha, \ell} J_{\alpha}^{\ell}(s),
$$


for some $P_{\ell}(x, y) \in \mathbb{R}[x, y], \kappa_{\alpha, \ell}:=\ell !\left(\begin{array}{l}\alpha \\ \ell\end{array}\right)$ and

$$
J_{\alpha}^{\ell}(s):=\int_{0}^{1-\phi(s)} g^{\alpha-\ell}(x ; s) \log ^{\ell}(1-x) d x .
$$

Since $\phi(s)$ is a flat function at $s=0$, it is clear that so it is the first summand in $\partial_{s}^{\ell} J_{\alpha}(s)$. Taking this into account, it is clear that the result will follow once we prove that

$$
\lim _{s \rightarrow 0^{+}} s^{\ell} J_{\alpha}^{\ell}(s)=\left\{\begin{array}{cl}
\frac{1}{1+\alpha} & \text { if } \ell=0 \\
0 & \text { if } \ell>0
\end{array}\right.
$$

With this aim in view we first note that, for all $\ell \geqslant 0$,

$$
\left|s^{\ell} J_{\alpha}^{\ell}(s)\right| \leqslant c_{\bullet}^{\alpha-\ell}(s) s^{\ell} \int_{0}^{1-s} x^{\alpha}\left(\frac{-\log (1-x)}{x}\right)^{\ell} d x+s^{\ell} \int_{1-s}^{1-\phi(s)} g^{\alpha-\ell}(x ; s)(-\log (1-x))^{\ell} d x
$$

where we set $c_{\bullet}=c_{+}$if $\alpha-\ell>0$ and $c_{\bullet}=c_{-}$otherwise. Here we use that $1-s<1-\varphi(s)$, due to $\lim _{s \rightarrow 0^{+}} \frac{\varphi(s)}{s}=0$, and that consequently $c_{-}(s) x \leqslant g(x ; s) \leqslant c_{+}(s) x$ for all $x \in(0,1-s)$. From the above inequality, using in the first summand the fact that $x \longmapsto \frac{-\log (1-x)}{x}$ is increasing and applying the Cauchy-Schwartz inequality in the second one, we get

$$
\left|s^{\ell} J_{\alpha}^{\ell}(s)\right| \leqslant c_{\bullet}^{\alpha-\ell}(s)\left(\frac{-s \log s}{1-s}\right)^{\ell} \frac{(1-s)^{\alpha+1}}{\alpha+1}+s^{\ell} L_{1}(s)^{1 / 2} L_{2}(s)^{1 / 2},
$$

with

$$
L_{1}(s):=\int_{1-s}^{1-\phi(s)} g^{2(\alpha-\ell)}(x ; s) d x \text { and } L_{2}(s):=\int_{1-s}^{1-\phi(s)}(-\log (1-x))^{2 \ell} d x
$$

One can verify that a primitive of $(-\log (1-x))^{2 \ell}$ is $(x-1) Q_{2 \ell}(\log (1-x))$, where $Q_{2 \ell}$ is a polynomial of degree $2 \ell$ with integer coefficients. On account of this we can assert that $L_{2}(s)^{1 / 2}$ tends to zero as $s \longrightarrow 0^{+}$. Let us study next the behaviour of $s^{\ell} L_{1}(s)^{1 / 2}$. If $\beta:=2(\alpha-\ell) \geqslant 0$ then $g^{\beta}(x ; s) \leqslant 1$, so that $L_{1}(s)$ tends to zero as $s \longrightarrow 0^{+}$. If $\beta<0$ then $g^{\beta}(x ; s) \leqslant m^{\beta}(x ; s)$ for $x \in[1-s, 1-\phi(s)]$ and

$$
L_{1}(s) \leqslant \int_{1-s}^{1-\phi(s)} m^{\beta}(x ; s) d x=\int_{0}^{s-\phi(s)}\left(\frac{\omega(s)}{s} u+s-\phi(s)\right)^{\beta} d u .
$$

Thus, by means of an easy integration, if $\beta \neq-1$ then we get

$$
L_{1}(s) \leqslant \frac{1}{\beta+1} \frac{s}{\omega(s)}\left((1-\phi(s) / s)^{\beta+1}(\omega(s)+s)^{\beta+1}-s^{\beta+1}(1-\phi(s) / s)^{\beta+1}\right) \leqslant C s^{\min (\beta+2,1)}
$$

for some constant $C>0$. (Here we use that $\lim _{s \rightarrow 0^{+}} \omega(s)=1$.) Consequently $s^{\ell} L_{1}(s)^{1 / 2} \leqslant C s^{\min \left(\alpha+1, \ell+\frac{1}{2}\right)}$, which tends to zero as $s \longrightarrow 0^{+}$. Likewise, if $\beta=-1$ then

$$
L_{1}(s) \leqslant \frac{s}{\omega(s)} \log \left(1+\frac{\omega(s)}{s}\right)
$$

which also tends to zero as $s \longrightarrow 0^{+}$. So far we have proved that

$$
\lim _{s \rightarrow 0^{+}} s^{\ell} L_{1}(s)^{1 / 2} L_{2}(s)^{1 / 2}=0 \text { for all } \ell \geqslant 0 .
$$

Taking this into account, from (3) we can assert that if $\ell>0$ then $\lim _{s \rightarrow 0^{+}} s^{\ell} J_{\alpha}^{\ell}(s)=0$, as desired. 
It only remains to be proved the assertion for $\ell=0$, i.e. that $\lim _{s \rightarrow 0^{+}} J_{\alpha}(s)=\frac{1}{1+\alpha}$. To this end we claim that $N_{-}(s) \leqslant J_{\alpha}(s) \leqslant N_{+}(s)$ for some functions with $\lim _{s \rightarrow 0^{+}} N_{ \pm}(s)=\frac{1}{1+\alpha}$. The existence of $N_{+}$follows from taking $\ell=0$ in (3) and (4) and the fact that $\lim _{s \rightarrow 0^{+}} c_{\bullet}(s)=1$. Let us show the existence of $N_{-}$. Setting $c_{\star}=c_{+}$if $\alpha \in(-1,0)$ and $c_{\star}=c_{-}$if $\alpha \geqslant 0$, we get that

$$
J_{\alpha}(s) \geqslant \int_{0}^{1-s} g^{\alpha}(x ; s) d x \geqslant c_{\star}^{\alpha}(s) \int_{0}^{1-s} x^{\alpha} d x=\frac{1}{\alpha+1} c_{\star}^{\alpha}(s)(1-s)^{\alpha+1}=: N_{-}(s) .
$$

Once again in the second inequality above we use the piecewise linear bounds and take $1-s<1-\varphi(s)$ into account. Since it is clear that $\lim _{s \rightarrow 0^{+}} N_{-}(s)=\frac{1}{\alpha+1}$, the claim follows and this completes the proof of the result.

Definition 2.5. Let $f_{0}, f_{1}, \ldots, f_{k-1}$ be analytic functions on an open interval $I$ of $\mathbb{R}$. Then

$$
W\left[f_{0}, f_{1}, \ldots, f_{k-1}\right](x)=\operatorname{det}\left(f_{j}^{(i)}(x)\right)_{0 \leq i, j \leq k-1}=\left|\begin{array}{ccc}
f_{0}(x) & \cdots & f_{k-1}(x) \\
f_{0}^{\prime}(x) & \cdots & f_{k-1}^{\prime}(x) \\
& \vdots & \\
f_{0}^{(k-1)}(x) & \cdots & f_{k-1}^{(k-1)}(x)
\end{array}\right|
$$

is the Wronskian of $\left(f_{0}, f_{1}, \ldots, f_{k-1}\right)$ at $x \in I$.

The following well-known result (see for instance $[9,10]$ ) will constitute the last ingredient in the proof of Theorem A.

Lemma 2.6. $\left(f_{0}, f_{1}, \ldots, f_{n-1}\right)$ is an ECT-system on $I$ if and only if, for each $k=1,2, \ldots, n$,

$$
W\left[f_{0}, f_{1}, \ldots, f_{k-1}\right](x) \neq 0 \text { for all } x \in I .
$$

Proof of Theorem A. As we already mentioned, we shall prove that for each $n \geqslant 0$ there exists $\varepsilon>0$ such that $\left(\hat{I}_{0}, \hat{I}_{1}, \ldots, \hat{I}_{n}\right)$ is an ECT-system on $(0, \varepsilon)$, where recall that $\hat{I}_{k}(s):=I_{k}(f(1 / s))$ with $f(x)=e^{-x}(1+x)$. This is equivalent to the assertion we want to prove because $s \longmapsto f(1 / s)$ is a diffeomorphism from $(0,+\infty)$ to $(0,1)$ with $\lim _{s \rightarrow 0^{+}} f(1 / s)=0$. Following the notation we have introduced so far, see $(1)$,

$$
\hat{I}_{k}(s)=2^{k+\frac{1}{2}} \hat{I}_{k}^{-}(s)+2^{k+\frac{1}{2}} \hat{I}_{k}^{+}(s), \text { where } \hat{I}_{k}^{ \pm}(s)=I_{k}^{ \pm}(f(1 / s)) .
$$

Since $I_{k}^{-}(h)$ is analytic at $h=0$ and $s \longmapsto f(1 / s)$ is flat at $s=0$, we can assert that $\hat{I}_{k}^{-}(s)=a_{k}+N_{k}(s)$, where $a_{k}$ is a constant and $N_{k}$ is a flat function. Thus Lemma 2.2 shows that

$$
\hat{I}_{k}(s)=(2 / s)^{k+\frac{1}{2}}\left(s^{k+\frac{1}{2}}\left(a_{k}+N_{k}(s)\right)+\psi_{k-\frac{1}{2}}(s)\right) .
$$

Then, by applying Lemma 2.3 and an easy computation, we can assert that

$$
\hat{I}_{k}(s)=(2 / s)^{k+\frac{1}{2}}\left(s^{k+\frac{1}{2}}\left(\hat{a}_{k}+\hat{N}_{k}(s)\right)+(1+s)^{k+\frac{1}{2}} J_{k-\frac{1}{2}}\left(\frac{s}{1+s}\right)\right)
$$

where again $\hat{a}_{k}$ is a constant and $\hat{N}_{k}$ is a flat function. The combination of this with Lemma 2.4 shows that we can write $\hat{I}_{k}(s)=(2 / s)^{k+\frac{1}{2}} L_{k}(s)$ with

$$
\lim _{s \rightarrow 0^{+}} s^{\ell} \partial_{s}^{\ell} L_{k}(s)=\left\{\begin{array}{cl}
\frac{1}{k+\frac{1}{2}} & \text { if } \ell=0 \\
0 & \text { if } \ell>0
\end{array}\right.
$$


Observe that $\left(\hat{I}_{k}(s)\right)_{k=0}^{n}$ is an ECT-system on $(0, \varepsilon)$ if, and only if, $\left(s^{n-k} L_{k}(s)\right)_{k=0}^{n}$ is an ECT-system on $(0, \varepsilon)$. (This follows by multiplying each function in the first system by $s^{n-\frac{1}{2}}$.) Thanks to Lemma 2.6 , the latter assertion is equivalent to show that $W_{i}(s) \neq 0$ for all $s \in(0, \varepsilon)$ and $i=0,1, \ldots, n$, where

$$
W_{i}(s):=W\left(L_{i}(s), s L_{i-1}(s), \ldots, s^{i} L_{0}(s)\right)=\left|\begin{array}{cccc}
L_{i}(s) & s L_{i-1}(s) & \cdots & s^{i} L_{0}(s) \\
\partial_{s} L_{i}(s) & \partial_{s}\left(s L_{i-1}(s)\right) & \cdots & \partial_{s}\left(s^{i} L_{0}(s)\right) \\
\vdots & \vdots & \ddots & \vdots \\
\partial_{s}^{i} L_{i}(s) & \partial_{s}^{i}\left(s L_{i-1}(s)\right) & \cdots & \partial_{s}^{i}\left(s^{i} L_{0}(s)\right)
\end{array}\right| .
$$

For all $k \in\{1,2, \ldots, i+1\}$, we multiply the $k$ th row by $s^{k}$ and the $k$ th column by $s^{-k}$. It is clear that the value of the determinant remains unchanged after these transformations. In doing so the $(\ell, k)$-entry of the corresponding matrix is equal to

$$
s^{\ell-k} \partial_{s}^{\ell}\left(s^{k} L_{i-k}(s)\right)=s^{\ell-k} \sum_{j=0}^{\ell}\left(\begin{array}{l}
\ell \\
j
\end{array}\right) \partial_{s}^{j}\left(s^{k}\right) \partial_{s}^{\ell-j}\left(L_{i-k}(s)\right)=\sum_{j=0}^{\ell}\left(\begin{array}{l}
\ell \\
j
\end{array}\right)\left(\begin{array}{l}
k \\
j
\end{array}\right) j ! s^{\ell-j} \partial_{s}^{\ell-j}\left(L_{i-k}(s)\right),
$$

which tends to $\left(\begin{array}{l}k \\ \ell\end{array}\right) \frac{\ell !}{i-k+\frac{1}{2}}$ as $s \longrightarrow 0^{+}$thanks to $(5)$. Since $\left(\begin{array}{c}k \\ \ell\end{array}\right)=0$ in case that $\ell>k$, the limit as $s \longrightarrow 0^{+}$ of the corresponding matrix is upper triangular and $\lim _{s \rightarrow 0^{+}} W_{i}(s)=\prod_{k=0}^{i} \frac{k !}{i-k+\frac{1}{2}} \neq 0$. Hence there exists $\varepsilon>0$ such that $W_{i}(s) \neq 0$ for $s \in(0, \varepsilon)$ and $i=0,1, \ldots, n$. This proves the validity of the result.

Corollary 2.7. For each $n \geqslant 0$ there exists a non-negative integer $k=k(n)$ such that any nontrivial linear combination

$$
\alpha_{0} I_{0}(h)+\alpha_{1} I_{1}(h)+\cdots+\alpha_{n-1} I_{n-1}(h)
$$

has at most $n+k-1$ isolated zeros on $(0,1]$ counted with multiplicities.

Proof. For the sake of shortness, given an analytic function $f$ on an interval $L$ we denote by $\mathcal{N}_{L}(f)$ the number of zeros of $f$ in $L$ counted according to multiplicity. Note that $\mathcal{N}_{L}(f)$ is a non-negative integer if $L$ is a closed interval and that it may be infinity otherwise. By Theorem A, there exists $\varepsilon>0$ such that $\left(I_{0}, I_{1}, \ldots, I_{n}\right)$ is an ECT-system on $(0, \varepsilon)$. Accordingly $\mathcal{N}_{(0, \varepsilon)}(f) \leqslant n$ for any $f \in\left\langle I_{0}, I_{1}, \ldots, I_{n}\right\rangle$. Therefore $\mathcal{N}_{(0,1]}(f) \leqslant n+\mathcal{N}_{[\varepsilon, 1]}(f)$ and, by applying [11, Theorem 2],

$$
\mathcal{N}_{[\varepsilon, 1]}(f) \leqslant n+\sum_{k=0}^{n} \mathcal{N}_{[\varepsilon, 1]}\left(W\left(I_{0}, \ldots, I_{k}\right)\right)+\sum_{k=0}^{n-2} \mathcal{N}_{[\varepsilon, 1]}\left(W\left(I_{0}, \ldots, I_{k}\right)\right) .
$$

This completes the proof of the result.

\section{References}

[1] V.I. Arnold, “Arnold's Problems", Springer-Verlag, Berlin, 2004.

[2] F. Dumortier, Chengzhi Li and Zifen Zhang, Unfolding of a quadratic integrable system with two centers and two unbounded heteroclinic loops, J. Differential Equations 139 (1997), 146-193.

[3] F. Dumortier and R. Roussarie, Abelian integrals and limit cycles, J. Differential Equations 227 (2006), 116-165.

[4] F. Dumortier and R. Roussarie, Birth of canard cycles, Discrete Contin. Dyn. Syst. 2 (2009), 723-781. 
[5] J-L. Figueras, W. Tucker and J. Villadelprat, Computer-assisted techniques for the verification of the Chebyshev property of Abelian integrals, J. Differential Equations 254 (2013), 3647-3663.

[6] J-P. Françoise and D. Xiao, Perturbation theory of a symmetric center within Liénard equations, J. DifferentialEquations 259 (2015), 2408-2429.

[7] M. Grau, F. Mañosas and J. Villadelprat, A Chebyshev criterion for Abelian integrals, Trans. Amer. Math. Soc. 363 (2011), 109-129.

[8] M. Han, Asymptotic expansions of Melnikov functions and limit cycle bifurcations, Internat. J. Bifur. Chaos Appl. Sci. Engrg. 22 (2012), 1250296.

[9] S. Karlin and W. Studden, "Tchebycheff systems: with applications in analysis and statistics", Interscience Publishers, 1966.

[10] M. Mazure, Chebyshev spaces and Bernstein bases, Constr. Approx. 22 (2005), 347-363.

[11] M. Voorhoeve and A. J. van der Poorten, Wronskian determinants and the zeros of certain functions, Indag. Math. 37 (1975), 417-424. 